Research Article

\title{
Periodic Solution for a Max-Type Fuzzy Difference Equation
}

\author{
Changyou Wang $(\mathbb{D}$ and Jiahui Li \\ College of Applied Mathematics, Chengdu University of Information Technology, Chengdu 610225, China
}

Correspondence should be addressed to Changyou Wang; wangcy@cuit.edu.cn

Received 26 April 2020; Accepted 9 June 2020; Published 10 July 2020

Academic Editor: Yonghui Sun

Copyright ( $) 2020$ Changyou Wang and Jiahui Li. This is an open access article distributed under the Creative Commons Attribution License, which permits unrestricted use, distribution, and reproduction in any medium, provided the original work is properly cited.

\begin{abstract}
The paper is concerned with the dynamics behavior of positive solutions for the following max-type fuzzy difference equation system: $x_{n+1}=\max \left\{A / x_{n}, A / x_{n-1}, x_{n-2}\right\}, n=0,1,2, \ldots$, where $x_{n}$ is a sequence of positive fuzzy numbers, and the parameter $A$ and the initial conditions $x_{-2}, x_{-1}, x_{0}$ are also positive fuzzy numbers. Firstly, the fuzzy set theory is used to transform the fuzzy difference equation into the corresponding ordinary difference equations with parameters. Then, the expression for the periodic solution of the max-type ordinary difference equations is obtained by the iteration, the inequality technique, and the mathematical induction. Moreover, we can obtain the expression for the periodic solution of the max-type fuzzy difference equation. In addition, the boundedness and persistence of solutions for the fuzzy difference equation is proved. Finally, the results of this paper are simulated and verified by using MATLAB 2016 software package.
\end{abstract}

\section{Introduction}

With the continuous development of science and technology in the fields of economy, biology, computer science, and so on, the research of nonlinear difference equations has been rapidly pushed forward, (for example, see [1-4] and the relevant reference cited therein). In the recent years, because the max operator has a great importance in automatic control models (see $[5,6]$ ), max-type difference equations which are a special type of difference equations have aroused the concern and attention of many scholars. The present max-type difference equations have evolved into a diverse family of the equation (for example, see [7-10] and the references cited therein).

Making a historical flash back for the max-type difference equation we study in this paper, we should mention that, in 2002, Voulov [11] studied positive solutions of the following equation:

$$
x_{n}=\max \left\{\frac{A}{x_{n-k}}, \frac{B}{x_{n-m}}\right\}, \quad n=0,1, \ldots,
$$

where $A$ and $B$ are any positive real numbers, $k$ and $m$ are any positive integers, and the initial values $x_{-1}, x_{-2}, \ldots, x_{-d}$, with $d=\max \{k, m\}$, are any positive numbers. This paper proved that every positive solution is eventually periodic with period $2 k$ if either $A>B$ or $A=B$ and $m=3 k$, with period $2 m$ if either $A<B$ or $A=B$ and $k=3 m$, and with period $k+m$ if $A=B$ and neither $m=3 k$ nor $m=3 k$. In 1998, Amleh et al. [12] showed that every well-defined positive solution of equation (1) is eventually periodic with period two, three, or four, when $k=1, m=2$, and $A=1$. In 2002, Mishev et al. [13] investigated, also, that every solution of equation (1) is eventually periodic, when $k=1, m=3$.

Recently, a number of scholars have made further research about equation (1). In 2010, Gelisken et al. [14] studied the following max-type difference equation:

$$
x_{n}=\max \left\{\frac{A}{x_{n-p}^{\alpha}}, \frac{B}{x_{n-k}^{\beta}}\right\}, \quad n=0,1, \ldots,
$$

where $p$ and $k$ are positive integers, $0<\alpha, \beta<1$, and $A, B>0$. They proved that every positive solution converges to $\bar{x}=\max \left\{A^{1 /(1+\alpha)}, B^{1 /(1+\beta)}\right\}$. In 2006, Yang et al. [15] proved that every positive solution of equation (2) converges to $\bar{x}=$ 1 or, eventually, periodic with period 4 , when $p=1, k=$ $2, \beta=1$ and $A=1$. In 2008, Sun [16] showed that every positive solution of equation (2) converges to 
$\bar{x}=\max \left\{A^{1 /(1+\alpha)}, B^{1 /(1+\beta)}\right\}$, when $p=1, k=2$. In 2009, Gelisken [17] proved that every positive solution of equation (2) converges to $\bar{x}=1$ or, eventually, periodic with period 2, when $p=1, k=3, \alpha=1$, and $B=1$. In addition, in 1999, Szalkai [18] studied the periodicity of solutions of the following difference equation:

$$
x_{n+1}=\max \left\{\frac{A}{x_{n}}, \frac{A}{x_{n-1}}, \cdots, \frac{A}{x_{n-k}}\right\}, \quad n=0,1, \ldots,
$$

where $k$ is a positive integer, $A$ is a real constant, and $\left\{x_{i}\right\}_{i=-k}^{0}$ are real numbers. He proved that every positive solution of (3) is eventually periodic of period $k+2$. In 2007, Bidwell and Franke [19] extended the form of equation (3) and considered the following difference equation:

$$
x_{n}=\max \left\{\frac{A_{n-1}^{1}}{x_{n-1}}, \frac{A_{n-1}^{2}}{x_{n-2}}, \cdots, \frac{A_{n-1}^{t}}{x_{n-t}}\right\}, \quad n=1,2, \ldots,
$$

where $t \in\{1,2, \ldots\},\left\{A_{n}^{i}\right\}_{n=0}^{\infty}$, for all $i=1,2, \ldots, t$, is a nonnegative periodic sequence with period $p_{i} \in z^{+}$, and initial conditions are positive numbers. They have showed that if every solution of (4) is bounded, then every solution is eventually periodic. In 2013, Cranston and Kent [20] extended some results obtained by Bidwell in equation (4), and they give both sufficient conditions on the $p_{i}^{\prime} s$ for the boundedness of all solutions and sufficient conditions for all solutions to be unbounded.

It is easy to see that the abovementioned equations are the same type difference equation. Now let us look at another type of equation. In 2012, Qin et al. [21] studied the following max-type difference equation:

$$
x_{n+1}=\max \left\{\frac{A}{x_{n}}, x_{n-k}\right\}, \quad n=0,1, \ldots,
$$

where $k$ is a positive integer, $A$ is a real constant, and the initial conditions $\left\{x_{i}\right\}_{i=-k}^{0}$ are real numbers. They show every well-defined solution of this equation is eventually periodic with period $k+1$. In 2009, Elsayed and Stević [22] considered equation (5), when $k=2$, and they showed that every well-defined solution of equation (5) is eventually periodic with period 3. In 2010, Iričanin and Elsayed [23] investigated equation (5), when $k=3$, and proved that every well-defined solution is eventually periodic with period 4. Recently, Xiao and Shi [24] studied equation (5), when $k=1$, and the result that every well-defined solution is eventually periodic with period 2 is obtained. Because of the necessity for some techniques, in 2014, Sun et al. [25] considered the following max-type equation that extends the form of equation (5):

$$
x_{n}=\max \left\{\frac{A_{n}}{x_{n-r}}, x_{n-k}\right\}, \quad n=0,1, \ldots,
$$

where $\left\{A_{n}\right\}_{n=1}^{+\infty}$ is a periodic sequence with period $p$ and $k, r \in\{1,2, \ldots\}$ with $\operatorname{gcd}\{k, r\}=1$ and $k \neq r$ and the initial conditions $\left\{x_{i}\right\}_{i=-d}^{0}$ are real numbers with $d=\max \{r, k\}$. They showed that if $p=1$ (or $p \geq 2$ and $k$ is odd), then every well-defined solution of this equation is eventually periodic with period $k$. So far, there are more studies on this kind of difference equations (for example, see [26-30] and the relevant reference cited therein).

With the development of the theory of difference equations, it is found that the given information needed to describe many practical problems in a difference equation model is incomplete. One of the effective procedures for considering uncertainty and imprecise real phenomena is the fuzzification of the corresponding difference equation systems. A numbers of studies have been made on the fuzzy difference equations so far, (for example, see [31-41] and the relevant reference cited therein). In recent decades, the maxtype fuzzy difference equations have been attracting great increasing attention. In 2004, Stefanidou and Papaschinopoulos [42] extended equation (3) from real number to fuzzy number, where $A$ is a positive fuzzy number and the initial conditions $\left\{x_{i}\right\}_{i=-k}^{0}$ are positive fuzzy numbers, and they gave a condition so that the solution is eventually periodic, unbounded, and nonpersistent and considered and studied the corresponding fuzzy difference equation (9) in [42] when $k=0, m=1$, i.e.,

$$
x_{n+1}=\max \left\{\frac{A_{0}}{x_{n}}, \frac{A_{1}}{x_{n-1}}\right\}, \quad n=0,1, \ldots,
$$

where $A_{0}$ and $A_{1}$ are positive fuzzy numbers and the initial values $x_{-1}$ and $x_{0}$ are any positive fuzzy numbers, and they proved the positive fuzzy solutions of equation (7) is eventually periodic, unbounded, and nonpersistent.

In 2006, Stefanidou and Papaschinopoulos [43], to further extend the difference equation (7), considered the periodic nature of the positive solutions of the following fuzzy difference equation:

$$
x_{n+1}=\max \left\{\frac{A_{0}}{x_{n-k}}, \frac{A_{1}}{x_{n-m}}\right\}, \quad n=0,1, \ldots,
$$

where $A_{0}$ and $A_{1}$ are positive fuzzy numbers and the initial values $x_{-d}, \ldots, x_{0}, d=\max \{k, m\}$ are any positive fuzzy numbers. Some latest related research can be found in [44-49].

The fuzzy difference equation we study in this paper is motivated by the abovementioned ordinary difference equation and corresponding fuzzy difference equation. More precisely, we consider the fuzzy difference equation of the following form:

$$
x_{n+1}=\max \left\{\frac{A}{x_{n}}, \frac{A}{x_{n-1}}, x_{n-2}\right\}, \quad n=0,1, \ldots,
$$

where $A$ are positive fuzzy numbers and the initial values $x_{-2}, x_{-1}, x_{0}$ are any positive fuzzy numbers. This paper aims to study the periodicity of the positive solutions of (9) by using a new iteration method for the more general nonlinear difference equations and inequality skills, as well as the mathematical induction.

\section{Preliminaries and Notations}

In order to facilitate the description of this paper, we need the following definitions. 
Definition 1 . For a set $B$, we denote by $\bar{B}$, the closure of $B$. We say that a function $A: R \longrightarrow[0,1]$ is a fuzzy number if the following conditions hold:

(i) $A$ is normal, i.e., there exists $x \in R$ such that $A(x)=1$

(ii) $A$ is a fuzzy convex, i.e., $A\left(t x_{1}+(1-t)\right.$ $\left.x_{2}\right) \geq \min \left\{A\left(x_{1}\right), A\left(x_{2}\right)\right\}, \forall t \in[0,1], x_{1}, x_{2} \in R$

(iii) $A$ is upper semicontinuous on $R$

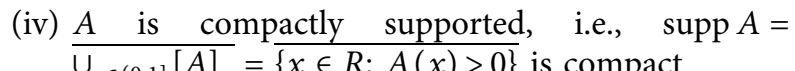

For $\alpha \in(0,1]$, the $\alpha$-cuts of $A$ on $R$ is defined as $[A]_{\alpha}=\{x \in R: A(x) \geq \alpha\}$. It is clear that the $[A]_{\alpha}$ is a bounded closed interval in $R$, and we say that a fuzzy number $A$ is positive if supp $A \subset(0, \infty)$.

It is obvious that if $A$ is a positive real number, then $A$ is a positive fuzzy number and $[A]_{\alpha}=[A, A], \alpha \in(0,1]$. At this time, we say that $A$ is a trivial fuzzy number.

Definition 2 ([32]). The fuzzy analog of the boundedness and persistence is as follows:

(i) A sequence of positive fuzzy numbers $x_{n}$ persists (resp. is bounded) if there exists a positive real numbers $M(\operatorname{resp} . N)$ such that $\operatorname{supp} x_{n} \epsilon$ $[M, \infty)\left(\right.$ resp. supp $\left.x_{n} \in[0, N)\right), n=1,2, \ldots$

(ii) A sequence of positive fuzzy numbers $x_{n}$ is bounded and persists if there exist positive real numbers $M, N>0$ such that $\operatorname{supp} x_{n} \in[M, N), n=1,2, \ldots$

\section{Main Results}

Firstly, we will prove a simple auxiliary result which will be used many times in the rest of the paper.

Lemma 1. Assume that $\left\{x_{n}\right\}_{n=-2}^{\infty}$ is a solution of equation (9), and there is $k_{0} \in N_{0} \cup\{-2,-1\}$ such that

$$
\begin{aligned}
x_{k_{0}} & =x_{k_{0}+3}, \\
x_{k_{0}+1} & =x_{k_{0}+4}, \\
x_{k_{0}+2} & =x_{k_{0}+5} .
\end{aligned}
$$

Then, this solution is eventually periodic with period three.

Proof. If the following equalities (11) are true, then (10) is true. Now, we need prove that

$$
\begin{aligned}
x_{k_{0}} & =x_{k_{0}+3 m}, \\
x_{k_{0}+1} & =x_{k_{0}+1+3 m}, \\
x_{k_{0}+2} & =x_{k_{0}+2+3 m},
\end{aligned}
$$

for every $m \in N$, from which the lemma follows.

We use the method of induction. For $m=1$, (11) becomes (10). Assume that (11) holds for $1 \leq m \leq m_{0}$. From this and by using (9) and (10), as well as an iterative method, one can obtain

$$
\begin{aligned}
x_{k_{0}+3\left(m_{0}+1\right)} & =\max \left\{\frac{A}{x_{k_{0}+3 m_{0}+2}}, \frac{A}{x_{k_{0}+3 m_{0}+1}}, x_{k_{0}+3 m_{0}}\right\} \\
& =\max \left\{\frac{A}{x_{k_{0}+2}}, \frac{A}{x_{k_{0}+1}}, x_{k_{0}}\right\}=x_{k_{0}+3}=x_{k_{0}}, \\
x_{k_{0}+1+3\left(m_{0}+1\right)} & =\max \left\{\frac{A}{x_{k_{0}+3 m_{0}+3}}, \frac{A}{x_{k_{0}+3 m_{0}+2}}, x_{k_{0}+3 m_{0}+1}\right\} \\
& =\max \left\{\frac{A}{x_{k_{0}+3}}, \frac{A}{x_{k_{0}+2}}, x_{k_{0}+1}\right\}=x_{k_{0}+4}=x_{k_{0}+1}, \\
x_{k_{0}+2+3\left(m_{0}+1\right)} & =\max \left\{\frac{A}{x_{k_{0}+3 m_{0}+4}}, \frac{A}{x_{k_{0}+3 m_{0}+3}}, x_{k_{0}+3 m_{0}+2}\right\} \\
& =\max \left\{\frac{A}{x_{k_{0}+4}}, \frac{A}{x_{k_{0}+3}}, x_{k_{0}+2}\right\}=x_{k_{0}+5}=x_{k_{0}+2} .
\end{aligned}
$$

The proof is completed.

Next, we will study the existence and uniqueness of the positive solutions of equation (9).

Theorem 1. Consider equation (9), and suppose that $A$ is a positive fuzzy number; then, for every positive fuzzy numbers $x_{-2}, x_{-1}, x_{0}$, there exists a unique positive solution $x_{n}$ of (9) with initial values $x_{-2}, x_{-1}, x_{0}$.

Proof. The proof is similar to Proposition 3.1 [42], so we omit the proof of Theorem 1 .

Now, we study the periodicity of the positive solutions of (9) when $A$ is a trivial fuzzy number. We need the following lemma.

Lemma 2. Consider the system of difference equations,

$$
\begin{aligned}
& y_{n+1}=\max \left\{\frac{A}{z_{n}}, \frac{A}{z_{n-1}}, y_{n-2}\right\}, \\
& z_{n+1}=\max \left\{\frac{A}{y_{n}}, \frac{A}{y_{n-1}}, z_{n-2}\right\}, \quad n \geq 0,
\end{aligned}
$$

where $A$ is positive real constant and the initial values $y_{i}, z_{i}, i=-2,-1,0$, are positive real numbers, and then, every positive solution of (13) is eventually periodic with period three.

Proof. Let $\left(y_{n}, z_{n}\right)$ be a positive solution of (12); we can obtain that

$$
\begin{aligned}
& y_{1}=\max \left\{\frac{A}{z_{0}}, \frac{A}{z_{-1}}, y_{-2}\right\}, \\
& z_{1}=\max \left\{\frac{A}{y_{0}}, \frac{A}{y_{-1}}, z_{-2}\right\},
\end{aligned}
$$


and thus, $y_{1} \geq A / z_{-1}$ and $z_{1} \geq A / y_{-1}$. It is easy to see that $\left\{y_{n}\right\}_{n=-2}^{\infty},\left\{z_{n}\right\}_{n=-2}^{\infty}$ are positive real constants, and then $z_{-1} \geq A / y_{1}$ and $y_{-1} \geq A / z_{1}$; therefore,

$$
\begin{aligned}
& y_{2}=\max \left\{\frac{A}{z_{1}}, \frac{A}{z_{0}}, y_{-1}\right\}=\max \left\{\frac{A}{z_{0}}, y_{-1}\right\}, \\
& z_{2}=\max \left\{\frac{A}{y_{1}}, \frac{A}{y_{0}}, z_{-1}\right\}=\max \left\{\frac{A}{y_{0}}, z_{-1}\right\} .
\end{aligned}
$$

From equation (13), if $n \geq 2$, then, we have

$$
y_{n} \geq \frac{A}{z_{n-2}}, y_{n-1} \geq \frac{A}{z_{n-2}} ; z_{n} \geq \frac{A}{y_{n-2}}, z_{n-1} \geq \frac{A}{y_{n-2}},
$$

and thus, we have

$$
z_{n-2} \geq \frac{A}{y_{n}}, z_{n-2} \geq \frac{A}{y_{n-1}} ; y_{n-2} \geq \frac{A}{z_{n}}, y_{n-2} \geq \frac{A}{z_{n-1}} \text {. }
$$

From (17), we have the following equations, when $n \geq 2$ :

$$
\begin{aligned}
& y_{n+1}=\max \left\{\frac{A}{z_{n}}, \frac{A}{z_{n-1}}, y_{n-2}\right\}=y_{n-2}, \\
& z_{n+1}=\max \left\{\frac{A}{y_{n}}, \frac{A}{y_{n-1}}, z_{n-2}\right\}=z_{n-2} .
\end{aligned}
$$

Hence,

$$
\begin{aligned}
y_{3 n} & =y_{0}, \\
y_{3 n+1} & =y_{1}, \\
y_{3 n+2} & =y_{2}, \\
z_{3 n} & =z_{0}, \\
z_{3 n+1} & =z_{1}, \\
z_{3 n+2} & =z_{2}, \quad n \geq 1 .
\end{aligned}
$$

We can find that the solution of equation (13) has the following form:

$$
\begin{aligned}
\begin{aligned}
& y=\left\{y_{0}, y_{1}, y_{2}, y_{3}, y_{4}, y_{5}, \ldots\right\}=\left\{y_{0}, y_{1}, y_{2}, y_{0}, y_{1}, y_{2}, \ldots\right\} \\
&=\left\{y_{0}, \max \left\{\frac{A}{z_{0}}, \frac{A}{z_{-1}}, y_{-2}\right\}, \max \left\{\frac{A}{z_{0}}, y_{-1}\right\}, y_{0}, \max \left\{\frac{A}{z_{0}}, \frac{A}{z_{-1}}, y_{-2}\right\}, \max \left\{\frac{A}{z_{0}}, y_{-1}\right\}, \ldots\right\}, \\
& z=\left\{z_{0}, z_{1}, z_{2}, z_{3}, z_{4}, z_{5}, \ldots\right\}=\left\{z_{0}, z_{1}, z_{2}, z_{0}, z_{1}, z_{2}, \ldots\right\} \\
&=\left\{z_{0}, \max \left\{\frac{A}{y_{0}}, \frac{A}{y_{-1}}, z_{-2}\right\}, \max \left\{\frac{A}{y_{0}}, z_{-1}\right\}, z_{0}, \max \left\{\frac{A}{y_{0}}, \frac{A}{y_{-1}}, z_{-2}\right\}, \max \left\{\frac{A}{y_{0}}, z_{-1}\right\}, \ldots\right\} . \\
& L_{3 n, \alpha}=L_{0, \alpha}, L_{3 n+1, \alpha}=L_{1, \alpha}, L_{3 n+2, \alpha}=L_{2, \alpha}, \\
& R_{3 n, \alpha}=R_{0, \alpha}, R_{3 n+1, \alpha}=R_{1, \alpha}, R_{3 n+2, \alpha}=R_{2, \alpha}, \quad n \geq 1, \alpha \in(0,1]
\end{aligned}
\end{aligned}
$$

Theorem 2. Consider equation (9) where A is a trivial fuzzy number and the initial values $x_{-2}, x_{-1}, x_{0}$ are positive fuzzy numbers. Then, every positive solution of (9) is eventually periodic with period three.

Proof. Let $x_{n}$ be a positive solution of (9) with initial values $x_{-2}, x_{-1}, x_{0}$, from $\alpha$-cuts; thus, we have

$$
\begin{aligned}
& {\left[x_{i}\right]_{\alpha}=\left[L_{i, \alpha}, R_{i, \alpha}\right], \quad i=-2,-1,0, \alpha \in(0,1],} \\
& {\left[x_{n}\right]_{\alpha}=\left[L_{n, \alpha}, R_{n, \alpha}\right], \quad i=1,2,3, \ldots, \alpha \in(0,1],}
\end{aligned}
$$

and $A$ is a trivial fuzzy number; thus,

$$
[A]_{\alpha}=\left[A_{l, \alpha}, A_{r, \alpha}\right]=[A, A], \quad \alpha \in(0,1] .
$$

From Theorem 1, $\left(L_{n, \alpha}, R_{n, \alpha}\right), i=1,2,3, \ldots, \alpha \in(0,1]$ satisfies system as follows:

$$
\begin{aligned}
& L_{n+1, \alpha}=\max \left\{\frac{A}{R_{n, \alpha}}, \frac{A}{R_{n-1, \alpha}}, L_{n-2, \alpha}\right\}, \\
& R_{n+1, \alpha}=\max \left\{\frac{A}{L_{n, \alpha}}, \frac{A}{L_{n-1, \alpha}}, R_{n-2, \alpha}\right\}, \quad n \geq 0 .
\end{aligned}
$$

Using Lemma 2, we have that
Therefore, we have that $x_{n}$ is eventually periodic of period three. The proof of Theorem 2 is completed.

In the following, we study the periodicity of the positive solutions of (9) when $A$ is a nontrivial fuzzy number. We need the following lemma.

Lemma 3. Consider the system of difference equations,

$$
\begin{aligned}
& y_{n+1}=\max \left\{\frac{B}{z_{n}}, \frac{B}{z_{n-1}}, y_{n-2}\right\}, \\
& z_{n+1}=\max \left\{\frac{C}{y_{n}}, \frac{C}{y_{n-1}}, z_{n-2}\right\}, \quad n \geq 0,
\end{aligned}
$$

where $B$ and $C$ are positive real constants and $B<C$, the initial values $y_{i}, z_{i}, i=-2,-1,0$ are positive real numbers, and then, every positive solution of (25) is eventually periodic with period three.

Proof. Let $\left(y_{n}, z_{n}\right)$ be a positive solution of (25), and we can obtain that 


$$
\begin{aligned}
& y_{1}=\max \left\{\frac{B}{z_{0}}, \frac{B}{z_{-1}}, y_{-2}\right\}, \\
& z_{1}=\max \left\{\frac{C}{y_{0}}, \frac{C}{y_{-1}}, z_{-2}\right\},
\end{aligned}
$$

and thus, $z_{1} \geq C / y_{-1} \geq B / y_{-1}$. It is easy to see that $\left\{y_{n}\right\}_{n=-2}^{\infty},\left\{z_{n}\right\}_{n=-2}^{\infty}$ are positive real constants, and then, $y_{-1} \geq B / z_{1}$; therefore,

$$
\begin{aligned}
& y_{2}=\max \left\{\frac{B}{z_{1}}, \frac{B}{z_{0}}, y_{-1}\right\}=\max \left\{\frac{B}{z_{0}}, y_{-1}\right\}, \\
& z_{2}=\max \left\{\frac{C}{y_{1}}, \frac{C}{y_{0}}, z_{-1}\right\} .
\end{aligned}
$$

From equation (25), if $n \geq 2$, then we have

$$
z_{n} \geq \frac{C}{y_{n-2}} \geq \frac{B}{y_{n-2}}, z_{n-1} \geq \frac{C}{y_{n-2}} \geq \frac{B}{y_{n-2}},
$$

and thus, we have

$$
y_{n-2} \geq \frac{B}{z_{n}}, y_{n-2} \geq \frac{B}{z_{n-1}} .
$$

From (29), if $n \geq 2$, we have

$$
y_{n+1}=\max \left\{\frac{B}{z_{n}}, \frac{B}{z_{n-1}}, y_{n-2}\right\}=y_{n-2} .
$$

Hence,

$$
\begin{aligned}
y_{3 n} & =y_{0}, \\
y_{3 n+1} & =y_{1}, \\
y_{3 n+2} & =y_{2}, \quad n \geq 1 .
\end{aligned}
$$

Now, we consider the period nature of $\left\{z_{n}\right\}_{n=3}^{\infty}$. There are three big cases to be considered.

$\left(a_{1}\right)$ Assume that $B / z_{0} \geq B / z_{-1}$ and $B / z_{0} \geq y_{-2}$, and then, $y_{1}=B / z_{0} .\left(a_{11}\right)$ Assume that $C / y_{0} \geq C / y_{-1}$ and $C / y_{0} \geq z_{-2}$, and then, $z_{1}=C / y_{0} .\left(a_{111}\right)$ Assume that $B / z_{0} \geq y_{-1}$, and then, $y_{2}=B / z_{0}$. Thus, we have

$$
\begin{aligned}
& z_{3}=\max \left\{\frac{C}{y_{2}}, \frac{C}{y_{1}}, z_{0}\right\}=\max \left\{\frac{C}{y_{1}}, \frac{C}{y_{1}}, \frac{B}{y_{1}}\right\}=\frac{C}{y_{1}}, \\
& z_{4}=\max \left\{\frac{C}{y_{3}}, \frac{C}{y_{2}}, z_{1}\right\}=\max \left\{\frac{C}{y_{0}}, \frac{C}{y_{1}}, \frac{C}{y_{0}}\right\}=\max \left\{\frac{C}{y_{1}}, \frac{C}{y_{0}}\right\}, \\
& z_{5}=\max \left\{\frac{C}{y_{4}}, \frac{C}{y_{3}}, z_{2}\right\}=\max \left\{\frac{C}{y_{1}}, \frac{C}{y_{0}}, z_{2}\right\}=z_{2}, \\
& z_{6}=\max \left\{\frac{C}{y_{5}}, \frac{C}{y_{4}}, z_{3}\right\}=\max \left\{\frac{C}{y_{2}}, \frac{C}{y_{1}}, z_{3}\right\}=z_{3}, \\
& z_{7}=\max \left\{\frac{C}{y_{6}}, \frac{C}{y_{5}}, z_{4}\right\}=\max \left\{\frac{C}{y_{0}}, \frac{C}{y_{2}}, z_{4}\right\}=z_{4}, \\
& z_{8}=\max \left\{\frac{C}{y_{7}}, \frac{C}{y_{6}}, z_{5}\right\}=\max \left\{\frac{C}{y_{1}}, \frac{C}{y_{0}}, z_{2}\right\}=z_{5} .
\end{aligned}
$$

Hence, $z_{6}=z_{3}, z_{7}=z_{4}$, and $z_{8}=z_{5}$. From this and by Lemma 1, from the induction and iterative method, we have

$$
\begin{aligned}
z_{3 n}=z_{3} & =\frac{C}{y_{1}}=\frac{C}{B} z_{0}, \\
z_{3 n+1}=z_{4} & =\max \left\{\frac{C}{B} z_{0}, \frac{C}{y_{0}}\right\}, \\
z_{3 n+2} & =z_{5}=\max \left\{\frac{C}{B} z_{0}, \frac{C}{y_{0}}, z_{-1}\right\}, \quad n \geq 2 .
\end{aligned}
$$

$\left(a_{112}\right)$ Assume that $y_{-1} \geq\left(B / z_{0}\right)$, and then $y_{2}=y_{-1}$; thus, we have

$$
\begin{aligned}
& z_{3}=\max \left\{\frac{C}{y_{2}}, \frac{C}{y_{1}}, z_{0}\right\}=\max \left\{\frac{C}{y_{-1}}, \frac{C}{y_{1}}, \frac{B}{y_{1}}\right\}=\frac{C}{y_{1}}, \\
& z_{4}=\max \left\{\frac{C}{y_{3}}, \frac{C}{y_{2}}, z_{1}\right\}=\max \left\{\frac{C}{y_{0}}, \frac{C}{y_{-1}}, z_{1}\right\}=z_{1}, \\
& z_{5}=\max \left\{\frac{C}{y_{4}}, \frac{C}{y_{3}}, z_{2}\right\}=\max \left\{\frac{C}{y_{1}}, \frac{C}{y_{0}}, z_{2}\right\}=z_{2}, \\
& z_{6}=\max \left\{\frac{C}{y_{5}}, \frac{C}{y_{4}}, z_{3}\right\}=\max \left\{\frac{C}{y_{2}}, \frac{C}{y_{1}}, z_{3}\right\}=z_{3}, \\
& z_{7}=\max \left\{\frac{C}{y_{6}}, \frac{C}{y_{5}}, z_{4}\right\}=\max \left\{\frac{C}{y_{0}}, \frac{C}{y_{-1}}, z_{4}\right\}=z_{4}, \\
& z_{8}=\max \left\{\frac{C}{y_{7}}, \frac{C}{y_{6}}, z_{5}\right\}=\max \left\{\frac{C}{y_{1}}, \frac{C}{y_{0}}, z_{2}\right\}=z_{5} .
\end{aligned}
$$

Hence, $z_{6}=z_{3}, z_{7}=z_{4}$, and $z_{8}=z_{5}$. From this and by Lemma 1, from the induction and iterative method, we have

$$
\begin{aligned}
z_{3 n} & =z_{3}=\frac{C}{B} z_{0}, \\
z_{3 n+1} & =z_{4}=\frac{C}{y_{0}}, \\
z_{3 n+2} & =z_{5}=\max \left\{\frac{C}{B} z_{0}, \frac{C}{y_{0}}, z_{-1}\right\}, \quad n \geq 2 .
\end{aligned}
$$

$\left(a_{12}\right)$ Assume that $C / y_{-1} \geq C / y_{0}$ and $C / y_{-1} \geq z_{-2}$, and then, $z_{1}=C / y_{-1}$. Thus, we have $(27)$ hold. $\left(a_{121}\right)$ Assume that $B / z_{0} \geq y_{-1}$, and then, $y_{2}=B / z_{0}$; thus, we find that $z_{3}, z_{5}, z_{6}, z_{7}$, and $z_{8}$ are the same with (32), and

$$
z_{4}=\max \left\{\frac{C}{y_{3}}, \frac{C}{y_{2}}, z_{1}\right\}=\max \left\{\frac{C}{y_{0}}, \frac{C}{y_{1}}, \frac{C}{y_{-1}}\right\}=\frac{C}{y_{-1}} .
$$

Hence, $z_{6}=z_{3}, z_{7}=z_{4}$, and $z_{8}=z_{5}$, From this and by Lemma 1, from the induction and iterative method, we have 


$$
\begin{aligned}
z_{3 n} & =z_{3}=\frac{C}{B} z_{0}, \\
z_{3 n+1} & =z_{4}=\frac{C}{y_{-1}}, \\
z_{3 n+2} & =z_{5}=\max \left\{\frac{C}{B} z_{0}, \frac{C}{y_{0}}, z_{-1}\right\}, \quad n \geq 2 .
\end{aligned}
$$

( $\left.a_{122}\right)$ Assume that $y_{-1} \geq B / z_{0}$, then $y_{2}=y_{-1}$; thus, we find that $z_{3}, z_{5}, z_{6}, z_{7}$, and $z_{8}$ are the same with (34), and

$$
z_{4}=\max \left\{\frac{C}{y_{3}}, \frac{C}{y_{2}}, z_{1}\right\}=\max \left\{\frac{C}{y_{0}}, \frac{C}{y_{-1}}, \frac{C}{y_{-1}}\right\}=\frac{C}{y_{-1}} \text {. }
$$

Hence, $z_{6}=z_{3}, z_{7}=z_{4}$, and $z_{8}=z_{5}$. From this and by Lemma 1, from the induction and iterative method, we have

$$
\begin{aligned}
z_{3 n} & =z_{3}=\frac{C}{B} z_{0}, \\
z_{3 n+1} & =z_{4}=\frac{C}{y_{-1}}, \\
z_{3 n+2} & =z_{5}=\max \left\{\frac{C}{B} z_{0}, \frac{C}{y_{0}}, z_{-1}\right\}, \quad n \geq 2 .
\end{aligned}
$$

( $\left.a_{13}\right)$ Assume that $z_{-2} \geq C / y_{0}$ and $z_{-2} \geq C / y_{-1}$, and then, $z_{1}=z_{-2}$. Thus, we have (27) hold. $\left(a_{131}\right)$ Assume that $B / z_{0} \geq y_{-1}$, and then, $y_{2}=B / z_{0}$; thus, we find that $z_{3}, z_{5}, z_{6}, z_{7}$, and $z_{8}$ are the same with (32), and

$$
z_{4}=\max \left\{\frac{C}{y_{3}}, \frac{C}{y_{2}}, z_{1}\right\}=\max \left\{\frac{C}{y_{0}}, \frac{C}{y_{1}}, z_{-2}\right\}=z_{-2} \text {. }
$$

Hence, $z_{6}=z_{3}, z_{7}=z_{4}$, and $z_{8}=z_{5}$. From this and by Lemma 1, from the induction and iterative method, we have

$$
\begin{aligned}
z_{3 n} & =z_{3}=\frac{C}{B} z_{0}, \\
z_{3 n+1}=z_{4} & =z_{-2}, \\
z_{3 n+2} & =z_{5}=\max \left\{\frac{C}{B} z_{0}, \frac{C}{y_{0}}, z_{-1}\right\}, \quad n \geq 2 .
\end{aligned}
$$

( $a_{132}$ ) Assume that $y_{-1} \geq B / z_{0}$, and then, $y_{2}=y_{-1}$; thus, we find that $z_{3}, z_{5}, z_{6}, z_{7}$, and $z_{8}$ are the same with (34), and

$$
z_{4}=\max \left\{\frac{C}{y_{3}}, \frac{C}{y_{2}}, z_{1}\right\}=\max \left\{\frac{C}{y_{0}}, \frac{C}{y_{-1}}, z_{-2}\right\}=z_{-2} \text {. }
$$

Hence, $z_{6}=z_{3}, z_{7}=z_{4}, z_{8}=z_{5}$. From this and by Lemma 1, from the induction and iterative method, we have

$$
\begin{aligned}
z_{3 n}=z_{3} & =\frac{C}{B} z_{0}, \\
z_{3 n+1}=z_{4} & =z_{-2}, \\
z_{3 n+2} & =z_{5}=\max \left\{\frac{C}{B} z_{0}, \frac{C}{y_{0}}, z_{-1}\right\}, \quad n \geq 2 .
\end{aligned}
$$

$\left(b_{1}\right)$ Assume that $B / z_{-1} \geq B / z_{0}$ and $B / z_{-1} \geq y_{-2}$, and then, $y_{1}=B / z_{-1}$. $\left(b_{11}\right)$ Assume that $C / y_{0} \geq C / y_{-1}$ and $C / y_{0} \geq z_{-2}$, and then, $z_{1}=C / y_{0}$. Thus, we have

$$
\begin{aligned}
& y_{2}=\max \left\{\frac{B}{z_{1}}, \frac{B}{z_{0}}, y_{-1}\right\}=\max \left\{\frac{B}{z_{0}}, y_{-1}\right\}, \\
& z_{2}=\max \left\{\frac{C}{y_{1}}, \frac{C}{y_{0}}, z_{-1}\right\}=\max \left\{\frac{C}{y_{1}}, \frac{C}{y_{0}}\right\}=\max \left\{\frac{C}{B} z_{-1}, \frac{C}{y_{0}}\right\} .
\end{aligned}
$$

$\left(b_{111}\right)$ Assume that $B / z_{0} \geq y_{-1}$, and then, $y_{2}=B / z_{0}$; thus, we find that $z_{3}, z_{5}, z_{6}, z_{7}$, and $z_{8}$ are the same with (32), and

$$
\begin{aligned}
& z_{3}=\max \left\{\frac{C}{y_{2}}, \frac{C}{y_{1}}, z_{0}\right\}=\max \left\{\frac{C}{y_{2}}, \frac{B}{y_{2}}\right\}=\frac{C}{y_{2}}=\frac{C}{B} z_{0}, \\
& z_{4}=\max \left\{\frac{C}{y_{3}}, \frac{C}{y_{2}}, z_{1}\right\}=\max \left\{\frac{C}{y_{0}}, \frac{C}{y_{2}}, \frac{C}{y_{0}}\right\}=\max \left\{\frac{C}{B} z_{0}, \frac{C}{y_{0}}\right\} .
\end{aligned}
$$

Hence, $z_{6}=z_{3}, z_{7}=z_{4}$, and $z_{8}=z_{5}$. From this and by Lemma 1, from the induction and iterative method, we have

$$
\begin{aligned}
z_{3 n} & =z_{3}=\frac{C}{B} z_{0}, \\
z_{3 n+1} & =z_{4}=\max \left\{\frac{C}{B} z_{0}, \frac{C}{y_{0}}\right\}, \\
z_{3 n+2} & =z_{5}=\max \left\{\frac{C}{B} z_{-1}, \frac{C}{y_{0}}\right\}, \quad n \geq 2 .
\end{aligned}
$$

$\left(b_{112}\right)$ Assume that $y_{-1} \geq B / z_{0}$, and then, $y_{2}=y_{-1}$; thus, we find that $z_{4}, z_{5}, z_{6}, z_{7}$, and $z_{8}$ are the same with (34), and

$$
z_{3}=\max \left\{\frac{C}{y_{2}}, \frac{C}{y_{1}}, z_{0}\right\}=\max \left\{\frac{C}{y_{-1}}, \frac{C}{B} z_{-1}, z_{0}\right\} .
$$

Hence, $z_{6}=z_{3}, z_{7}=z_{4}$, and $z_{8}=z_{5}$. From this and by Lemma 1, from the induction and iterative method, we have

$$
\begin{aligned}
z_{3 n} & =z_{3}=\max \left\{\frac{C}{y_{-1}}, \frac{C}{B} z_{-1}, z_{0}\right\}, \\
z_{3 n+1} & =z_{4}=\frac{C}{y_{0}}, \\
z_{3 n+2} & =z_{5}=\max \left\{\frac{C}{B} z_{-1}, \frac{C}{y_{0}}\right\}, \quad n \geq 2 .
\end{aligned}
$$

$\left(b_{12}\right)$ Assume that $C / y_{-1} \geq C / y_{0}$ and $C / y_{-1} \geq z_{-2}$, and then, $z_{1}=C / y_{-1}$. Thus, we have (44) hold. $\left(b_{121}\right)$ Assume that $B / z_{0} \geq y_{-1}$, and then, $y_{2}=B / z_{0}$; thus, we find that $z_{3}, z_{5}, z_{6}, z_{7}$, and $z_{8}$ are the same with (32), and

$$
\begin{aligned}
& z_{3}=\max \left\{\frac{C}{y_{2}}, \frac{C}{y_{1}}, z_{0}\right\}=\max \left\{\frac{C}{y_{2}}, \frac{C}{y_{1}}\right\}=\frac{C}{y_{2}}=\frac{C}{B} z_{0}, \\
& z_{4}=\max \left\{\frac{C}{y_{3}}, \frac{C}{y_{2}}, z_{1}\right\}=\max \left\{\frac{C}{y_{0}}, \frac{C}{y_{2}}, \frac{C}{y_{-1}}\right\}=\max \left\{\frac{C}{B} z_{0}, \frac{C}{y_{-1}}\right\} .
\end{aligned}
$$


Hence, $z_{6}=z_{3}, z_{7}=z_{4}$, and $z_{8}=z_{5}$. From this and by Lemma 1, from the induction and iterative method, we have

$$
\begin{aligned}
z_{3 n} & =z_{3}=\frac{C}{B} z_{0}, \\
z_{3 n+1} & =z_{4}=\max \left\{\frac{C}{B} z_{0}, \frac{C}{y_{-1}}\right\}, \\
z_{3 n+2} & =z_{5}=\max \left\{\frac{C}{B} z_{-1}, \frac{C}{y_{0}}\right\}, \quad n \geq 2 .
\end{aligned}
$$

$\left(b_{122}\right)$ Assume that $y_{-1} \geq B / z_{0}$, and then, $y_{2}=y_{-1}$; thus, we find that $z_{4}, z_{5}, z_{6}, z_{7}$, and $z_{8}$ are the same with (34), and

$$
z_{3}=\max \left\{\frac{C}{y_{2}}, \frac{C}{y_{1}}, z_{0}\right\}=\max \left\{\frac{C}{y_{-1}}, \frac{C}{B} z_{-1}, z_{0}\right\} .
$$

Hence, $z_{6}=z_{3}, z_{7}=z_{4}$, and $z_{8}=z_{5}$. From this and by Lemma 1 , from the induction and iterative method, we have

$$
\begin{aligned}
z_{3 n} & =z_{3}=\max \left\{\frac{C}{y_{-1}}, \frac{C}{B} z_{-1}, z_{0}\right\}, \\
z_{3 n+1} & =z_{4}=\frac{C}{y_{-1}} \\
z_{3 n+2} & =z_{5}=\max \left\{\frac{C}{B} z_{-1}, \frac{C}{y_{0}}\right\}, \quad n \geq 2 .
\end{aligned}
$$

$\left(b_{13}\right)$ Assume that $z_{-2} \geq C / y_{0}$ and $z_{-2} \geq C / y_{-1}$, and then, $z_{1}=z_{-2}$. Thus, we have (44) hold. $\left(b_{131}\right)$ Assume that $B / z_{0} \geq y_{-1}$, and then, $y_{2}=B / z_{0}$; thus, we can find that $z_{5}, z_{6}, z_{7}$, and $z_{8}$ are the same with (32), and

$$
\begin{aligned}
& z_{3}=\max \left\{\frac{C}{y_{2}}, \frac{C}{y_{1}}, z_{0}\right\}=\frac{C}{y_{2}}=\frac{C}{B} z_{0}, \\
& z_{4}=\max \left\{\frac{C}{y_{3}}, \frac{C}{y_{2}}, z_{1}\right\}=\max \left\{\frac{C}{y_{0}}, \frac{C}{B} z_{0}, z_{1}\right\}=\max \left\{\frac{C}{B} z_{0}, z_{-2}\right\} .
\end{aligned}
$$

Hence, $z_{6}=z_{3}, z_{7}=z_{4}$, and $z_{8}=z_{5}$. From this and by Lemma 1, from the induction and iterative method, we have

$$
\begin{aligned}
z_{3 n} & =z_{3}=\frac{C}{B} z_{0}, \\
z_{3 n+1}=z_{4} & =\max \left\{\frac{C}{B} z_{0}, z_{-2}\right\}, \\
z_{3 n+2} & =z_{5}=\max \left\{\frac{C}{B} z_{-1}, \frac{C}{y_{0}}\right\}, \quad n \geq 2 .
\end{aligned}
$$

$\left(b_{132}\right)$ Assume that $y_{-1} \geq B / z_{0}$, and then, $y_{2}=y_{-1}$; thus, we find that $z_{4}, z_{5}, z_{6}, z_{7}$, and $z_{8}$ are the same with (34), and

$$
z_{3}=\max \left\{\frac{C}{y_{2}}, \frac{C}{y_{1}}, z_{0}\right\}=\max \left\{\frac{C}{y_{-1}}, \frac{C}{B} z_{-1}, z_{0}\right\} \text {. }
$$

Hence, $z_{6}=z_{3}, z_{7}=z_{4}$, and $z_{8}=z_{5}$. From this and by Lemma 1, from the induction and iterative method, we have

$$
\begin{aligned}
z_{3 n} & =z_{3}=\max \left\{\frac{C}{y_{-1}}, \frac{C}{B} z_{-1}, z_{0}\right\}, \\
z_{3 n+1}=z_{4} & =z_{-2}, \\
z_{3 n+2} & =z_{5}=\max \left\{\frac{C}{B} z_{-1}, \frac{C}{y_{0}}\right\}, \quad n \geq 2 .
\end{aligned}
$$

$\left(c_{1}\right)$ Assume that $y_{-2} \geq B / z_{-1}$ and $y_{-2} \geq B / z_{0}$, and then, $y_{1}=y_{-2}$. $\left(c_{11}\right)$ Assume that $C / y_{0} \geq C / y_{-1}$ and $C / y_{0} \geq z_{-2}$, and then, $z_{1}=C / y_{0}$. Thus, we have

$$
\begin{aligned}
& y_{2}=\max \left\{\frac{B}{z_{1}}, \frac{B}{z_{0}}, y_{-1}\right\}=\max \left\{\frac{B}{z_{0}}, y_{-1}\right\}, \\
& z_{2}=\max \left\{\frac{C}{y_{1}}, \frac{C}{y_{0}}, z_{-1}\right\}=\max \left\{\frac{C}{y_{-2}}, \frac{C}{y_{0}}, z_{-1}\right\} .
\end{aligned}
$$

( $\left.c_{111}\right)$ Assume that $B / z_{0} \geq y_{-1}$, and then, $y_{2}=B / z_{0}$; thus, we find that $z_{5}, z_{6}, z_{7}$, and $z_{8}$ are the same with (32), and

$$
\begin{aligned}
& z_{3}=\max \left\{\frac{C}{y_{2}}, \frac{C}{y_{1}}, z_{0}\right\}=\max \left\{\frac{C}{y_{2}}, \frac{C}{y_{1}}\right\}=\max \left\{\frac{C}{B} z_{0}, \frac{C}{y_{-2}}\right\}, \\
& z_{4}=\max \left\{\frac{C}{y_{3}}, \frac{C}{y_{2}}, z_{1}\right\}=\max \left\{\frac{C}{y_{0}}, \frac{C}{y_{2}}, \frac{C}{y_{0}}\right\}=\max \left\{\frac{C}{B} z_{0}, \frac{C}{y_{0}}\right\} .
\end{aligned}
$$

Hence, $z_{6}=z_{3}, z_{7}=z_{4}$, and $z_{8}=z_{5}$. From this and by Lemma 1, from the induction and iterative method, we have

$$
\begin{aligned}
z_{3 n} & =z_{3}=\max \left\{\frac{C}{B} z_{0}, \frac{C}{y_{-2}}\right\}, \\
z_{3 n+1}=z_{4} & =\max \left\{\frac{C}{B} z_{0}, \frac{C}{y_{0}}\right\}, \\
z_{3 n+2} & =z_{5}=\max \left\{\frac{C}{y_{-2}}, \frac{C}{y_{0}}, z_{-1}\right\}, \quad n \geq 2 .
\end{aligned}
$$

( $c_{112}$ ) Assume that $y_{-1} \geq B / z_{0}$, and then, $y_{2}=y_{-1}$; thus, we find that $z_{4}, z_{5}, z_{6}, z_{7}$, and $z_{8}$ are the same with (34), and

$$
z_{3}=\max \left\{\frac{C}{y_{2}}, \frac{C}{y_{1}}, z_{0}\right\}=\max \left\{\frac{C}{y_{-1}}, \frac{C}{y_{-2}}, z_{0}\right\} \text {. }
$$

Hence, $z_{6}=z_{3}, z_{7}=z_{4}$, and $z_{8}=z_{5}$, From this and by Lemma 1, from the induction and iterative method, we have

$$
\begin{aligned}
z_{3 n} & =z_{3}=\max \left\{\frac{C}{y_{-1}}, \frac{C}{y_{-2}}, z_{0}\right\}, \\
z_{3 n+1} & =z_{4}=\frac{C}{y_{0}} \\
z_{3 n+2} & =z_{5}=\max \left\{\frac{C}{y_{-2}}, \frac{C}{y_{0}}, z_{-1}\right\}, \quad n \geq 2 .
\end{aligned}
$$

$\left(c_{12}\right)$ Assume that $C / y_{-1} \geq C / y_{0}$ and $C / y_{-1} \geq z_{-2}$, and then, $z_{1}=C / y_{-1}$. Thus, we have (57) hold. $\left(c_{121}\right)$ Assume 
that $B / z_{0} \geq y_{-1}$, and then, $y_{2}=B / z_{0}$; thus, we find that $z_{5}, z_{6}, z_{7}$, and $z_{8}$ are the same with (32), and

$$
\begin{aligned}
& z_{3}=\max \left\{\frac{C}{y_{2}}, \frac{C}{y_{1}}, z_{0}\right\}=\max \left\{\frac{C}{B} z_{0}, \frac{C}{y_{-2}}\right\}, \\
& z_{4}=\max \left\{\frac{C}{y_{3}}, \frac{C}{y_{2}}, z_{1}\right\}=\max \left\{\frac{C}{y_{2}}, \frac{C}{y_{-1}}\right\}=\max \left\{\frac{C}{B} z_{0}, \frac{C}{y_{-1}}\right\} .
\end{aligned}
$$

Hence, $z_{6}=z_{3}, z_{7}=z_{4}$, and $z_{8}=z_{5}$. From this and by Lemma 1, from the induction and iterative method, we have

$$
\begin{gathered}
z_{3 n}=z_{3}=\max \left\{\frac{C}{B} z_{0}, \frac{C}{y_{-2}}\right\}, \\
z_{3 n+1}=z_{4}=\max \left\{\frac{C}{B} z_{0}, \frac{C}{y_{-1}}\right\}, \\
z_{3 n+2}=z_{5}=\max \left\{\frac{C}{y_{-2}}, \frac{C}{y_{0}}, z_{-1}\right\}, \quad n \geq 2 .
\end{gathered}
$$

$\left(c_{122}\right)$ Assume that $y_{-1} \geq B / z_{0}$, and then, $y_{2}=y_{-1}$; thus, we find that $z_{5}, z_{6}, z_{7}$, and $z_{8}$ are the same with (34), and

$$
\begin{aligned}
& z_{3}=\max \left\{\frac{C}{y_{2}}, \frac{C}{y_{1}}, z_{0}\right\}=\max \left\{\frac{C}{y_{-1}}, \frac{C}{y_{1}}, z_{0}\right\}=\max \left\{\frac{C}{y_{-1}}, \frac{C}{y_{-2}}, z_{0}\right\}, \\
& z_{4}=\max \left\{\frac{C}{y_{3}}, \frac{C}{y_{2}}, z_{1}\right\}=\max \left\{\frac{C}{y_{0}}, \frac{C}{y_{-1}}, \frac{C}{y_{-1}}\right\}=\frac{C}{y_{-1}} .
\end{aligned}
$$

Hence, $z_{6}=z_{3}, z_{7}=z_{4}$, and $z_{8}=z_{5}$. From this and by Lemma 1, from the induction and iterative method, we have

$$
\begin{gathered}
z_{3 n}=z_{3}=\max \left\{\frac{C}{y_{-1}}, \frac{C}{y_{-2}}, z_{0}\right\}, \\
z_{3 n+1}=z_{4}=\frac{C}{y_{-1}} \\
z_{3 n+2}=z_{5}=\max \left\{\frac{C}{y_{-2}}, \frac{C}{y_{0}}, z_{-1}\right\}, \quad n \geq 2 .
\end{gathered}
$$

$\left(c_{13}\right)$ Assume that $z_{-2} \geq C / y_{0}$ and $z_{-2} \geq C / y_{-1}$, and then, $z_{1}=z_{-2}$. Thus, we have (57) hold. $\left(c_{131}\right)$ Assume that $B / z_{0} \geq y_{-1}$, and then, $y_{2}=B / z_{0}$; thus, we find that $z_{5}, z_{6}, z_{7}$, and $z_{8}$ are the same with (32), and

$$
\begin{aligned}
& z_{3}=\max \left\{\frac{C}{y_{2}}, \frac{C}{y_{1}}, z_{0}\right\}=\max \left\{\frac{C}{B} z_{0}, \frac{C}{y_{-2}}\right\}, \\
& z_{4}=\max \left\{\frac{C}{y_{3}}, \frac{C}{y_{2}}, z_{1}\right\}=\max \left\{\frac{C}{B} z_{0}, z_{-2}\right\} .
\end{aligned}
$$

Hence, $z_{6}=z_{3}, z_{7}=z_{4}$, and $z_{8}=z_{5}$, From this and by Lemma 1, from the induction and iterative method, we have

$$
\begin{aligned}
z_{3 n} & =z_{3}=\max \left\{\frac{C}{B} z_{0}, \frac{C}{y_{-2}}\right\}, \\
z_{3 n+1} & =z_{4}=\max \left\{\frac{C}{B} z_{0}, z_{-2}\right\}, \\
z_{3 n+2}=z_{5} & =\max \left\{\frac{C}{y_{-2}}, \frac{C}{y_{0}}, z_{-1}\right\}, \quad n \geq 2 .
\end{aligned}
$$

( $c_{132}$ ) Assume that $y_{-1} \geq B / z_{0}$, and then, $y_{2}=y_{-1}$; thus, we find that $z_{5}, z_{6}, z_{7}$, and $z_{8}$ are the same with (34), and

$$
z_{3}=\max \left\{\frac{C}{y_{2}}, \frac{C}{y_{1}}, z_{0}\right\}=\max \left\{\frac{C}{y_{-1}}, \frac{C}{y_{-2}}, z_{0}\right\} \text {. }
$$

Hence, $z_{6}=z_{3}, z_{7}=z_{4}$, and $z_{8}=z_{5}$. From this and by Lemma 1, from the induction and iterative method, we have

$$
\begin{aligned}
z_{3 n} & =z_{3}=\max \left\{\frac{C}{y_{-1}}, \frac{C}{y_{-2}}, z_{0}\right\}, \\
z_{3 n+1} & =z_{4}=z_{-2}, \\
z_{3 n+2} & =z_{5}=\max \left\{\frac{C}{y_{-2}}, \frac{C}{y_{0}}, z_{-1}\right\}, \quad n \geq 2 .
\end{aligned}
$$

The proof is complete.

Theorem 3. Consider equation (9) where $A$ is a nontrivial fuzzy number and the initial values $x_{-2}, x_{-1}, x_{0}$ are positive fuzzy numbers. Then, every positive solution of (9) is eventually periodic with period three.

Proof. Let $x_{n}$ be a positive solution of (9) with initial values $x_{-2}, x_{-1}, x_{0}$, such that we have (21) hold, and $A$ is a nontrivial fuzzy number; thus,

$$
[A]_{\alpha}=\left[A_{l, \alpha}, A_{r, \alpha}\right]=[B, C], \quad \alpha \in(0,1] .
$$

From Theorem 1, $\left(L_{n, \alpha}, R_{n, \alpha}\right), i=1,2,3, \ldots, \alpha \in(0,1]$ satisfies the system as follows:

$$
\begin{aligned}
& L_{n+1, \alpha}=\max \left\{\frac{B}{R_{n, \alpha}}, \frac{B}{R_{n-1, \alpha}}, L_{n-2, \alpha}\right\}, \\
& R_{n+1, \alpha}=\max \left\{\frac{C}{L_{n, \alpha}}, \frac{C}{L_{n-1, \alpha}}, R_{n-2, \alpha}\right\}, \quad n \geq 0 .
\end{aligned}
$$

Using Lemma 3, we have that

$$
\begin{aligned}
& L_{3 n, \alpha}=L_{3, \alpha}, L_{3 n+1, \alpha}=L_{4, \alpha}, L_{3 n+2, \alpha}=L_{5, \alpha}, \\
& R_{3 n, \alpha}=R_{3, \alpha}, R_{3 n+1, \alpha}=R_{4, \alpha}, R_{3 n+2, \alpha}=R_{5, \alpha}, \quad n \geq 2, \alpha \in(0,1] .
\end{aligned}
$$

Therefore, we have that $x_{n}$ is eventually periodic of period three. The proof is completed.

Theorem 4. Consider equation (9) where $A$ is a positive fuzzy number and the initial values $x_{-2}, x_{-1}, x_{0}$ are positive fuzzy numbers. Then, every positive solution of (9) is bounded and persists. 
Proof. From Theorem 3, $\left(L_{n, \alpha}, R_{n, \alpha}\right), i=1,2,3, \ldots, \alpha \in$ $(0,1]$ satisfies the system as follows:

$$
\begin{aligned}
& L_{n+1, \alpha}=\max \left\{\frac{A_{l, \alpha}}{R_{n, \alpha}}, \frac{A_{l, \alpha}}{R_{n-1, \alpha}}, L_{n-2, \alpha}\right\}, \\
& R_{n+1, \alpha}=\max \left\{\frac{A_{r, \alpha}}{L_{n, \alpha}}, \frac{A_{r, \alpha}}{L_{n-1, \alpha}}, R_{n-2, \alpha}\right\}, \quad n \geq 0,
\end{aligned}
$$

and

$$
\begin{aligned}
& L_{3 n, \alpha}=L_{3, \alpha}, L_{3 n+1, \alpha}=L_{4, \alpha}, L_{3 n+2, \alpha}=L_{5, \alpha} \\
& R_{3 n, \alpha}=R_{3, \alpha}, R_{3 n+1, \alpha}=R_{4, \alpha}, R_{3 n+2, \alpha}=R_{5, \alpha}, \quad n \geq 2, \alpha \in(0,1] .
\end{aligned}
$$

Form Lemma 3, we find $L_{n, \alpha}$ are equal to one of (i)-(iii):

(i) $L_{3 n, \alpha}=L_{0, \alpha}$

(ii) $L_{3 n+1, \alpha}=L_{1, \alpha}=\max \left\{A_{l, \alpha} / R_{0, \alpha}, A_{l, \alpha} / R_{-1, \alpha}, L_{-2, \alpha}\right\}$

(iii) $L_{3 n+2, \alpha}=L_{2, \alpha}=\max \left\{\min \left\{A_{l, \alpha} / A_{r, \alpha} L_{0, \alpha}, A_{l, \alpha} / A_{r, \alpha}\right.\right.$ $\left.\left.L_{-1, \alpha}, A_{l, \alpha} / R_{-2, \alpha}\right\}, A_{l, \alpha} / R_{0, \alpha}, L_{-1, \alpha}\right\}$

Thus, there exist numbers $M, N \in(0, \infty)$ such that

$$
\operatorname{supp} L_{n, \alpha} \in[M, N], \quad n=1,2,3, \ldots .
$$

Similarly, $R_{n, \alpha}$ are equal to one of $R_{3, \alpha}, R_{4, \alpha}$, and $R_{5, \alpha}$. From (33), (35)-(43), (46)-(56), and (59)-(69), we have

(1) $R_{3, \alpha}$ is equal to one of $\left(a_{i}\right)-\left(a_{i v}\right)$ :

$$
\begin{aligned}
& \left(a_{i}\right) \frac{A_{r, \alpha}}{A_{l, \alpha}} R_{0, \alpha},\left(a_{i i}\right) \max \left\{\frac{A_{r, \alpha}}{L_{-1, \alpha}}, \frac{A_{r, \alpha}}{A_{l, \alpha}} R_{-1, \alpha}, R_{0, \alpha}\right\}, \\
& \left(a_{i i i}\right) \max \left\{\frac{A_{r, \alpha}}{A_{l, \alpha}} R_{0, \alpha}, \frac{A_{r, \alpha}}{L_{-2, \alpha}}\right\},\left(a_{i v}\right) \max \left\{\frac{A_{r, \alpha}}{L_{-1, \alpha}}, \frac{A_{r, \alpha}}{L_{-2, \alpha}}, R_{0, \alpha}\right\} .
\end{aligned}
$$

(2) $R_{4, \alpha}$ is equal to one of $\left(b_{i}\right)-\left(b_{v i}\right)$ :

$\left(b_{i}\right) \frac{A_{r, \alpha}}{L_{0, \alpha}},\left(b_{i i}\right) \max \left\{\frac{A_{r, \alpha}}{A_{l, \alpha}} R_{0, \alpha}, \frac{A_{r, \alpha}}{L_{0, \alpha}}\right\},\left(b_{i i i}\right) \frac{A_{r, \alpha}}{L_{-1, \alpha}},\left(b_{i v}\right) R_{-2, \alpha}$,

$$
\left(b_{v}\right) \max \left\{\frac{A_{r, \alpha}}{A_{l, \alpha}} R_{0, \alpha}, \frac{A_{r, \alpha}}{L_{-1, \alpha}}\right\},\left(b_{v i}\right) \max \left\{\frac{A_{r, \alpha}}{A_{l, \alpha}} R_{0, \alpha}, R_{-2, \alpha}\right\} \text {. }
$$

(3) $R_{5, \alpha}$ is equal to one of $\left(c_{i}\right)-\left(c_{i i i}\right)$ :

$$
\begin{aligned}
& \left(c_{i}\right) \max \left\{\frac{A_{r, \alpha}}{A_{l, \alpha}} R_{0, \alpha}, \frac{A_{r, \alpha}}{L_{0, \alpha}}, R_{-1, \alpha}\right\},\left(c_{i i}\right) \max \left\{\frac{A_{r, \alpha}}{A_{l, \alpha}} R_{-1, \alpha}, \frac{A_{r, \alpha}}{L_{0, \alpha}}\right\}, \\
& \left(c_{i i i}\right) \max \left\{\frac{A_{r, \alpha}}{L_{-2, \alpha}}, \frac{A_{r, \alpha}}{L_{0, \alpha}}, R_{-1, \alpha}\right\} .
\end{aligned}
$$
that

Thus, there exist numbers $P, Q \in(0, \infty), P \geq N$ such

$$
\operatorname{supp} R_{n, \alpha} \in[P, Q], \quad n=1,2,3, \ldots
$$

From (75) and (79), for any $x_{n}$, there exists $\mu, \nu \in(0, \infty)$ such that

$$
\operatorname{supp} x_{n} \in[\mu, \nu]
$$

The proof is completed.

\section{An Illustrative Example}

To illustrate our results, we give an example in which the conditions of our theorem hold.

Example 1. Consider the following fuzzy difference equation:

$$
x_{n+1}=\max \left\{\frac{A}{x_{n}}, \frac{A}{x_{n-1}}, x_{n-2}\right\}, \quad n=0,1,2, \ldots,
$$

where $A$ is a positive fuzzy number. Moreover, these are defined in here by

$$
A(x)= \begin{cases}\frac{1}{2} x-\frac{3}{2}, & 3 \leq x \leq 5, \\ -\frac{1}{3} x+\frac{8}{3}, & 5 \leq x \leq 8 .\end{cases}
$$

In addition, we take the initial conditions $x_{-2}, x_{-1}, x_{0}$ such that

$$
x_{0}(x)= \begin{cases}x-4, & 4 \leq x \leq 5, \\ -\frac{1}{3} x+\frac{8}{3}, & 5 \leq x \leq 8 .\end{cases}
$$

$$
\begin{aligned}
& x_{-1}(x)= \begin{cases}\frac{1}{3} x-\frac{2}{3}, & 2 \leq x \leq 3.4, \\
-\frac{1}{2} x+\frac{7}{2}, & 3.4 \leq x \leq 7 .\end{cases} \\
& x_{-2}(x)= \begin{cases}x-\frac{3}{2}, & 1.5 \leq x \leq 2.5, \\
-2 x+6, & 2.5 \leq x \leq 3 .\end{cases}
\end{aligned}
$$

From (82), we get $[A]_{\alpha}=[3+2 \alpha, 8-3 \alpha], \quad \alpha \in(0,1]$. From (83), we get

$$
\begin{aligned}
{\left[x_{0}\right]_{\alpha} } & =[4+\alpha, 8-3 \alpha], \\
{\left[x_{-1}\right]_{\alpha} } & =[2+3 \alpha, 7-2 \alpha], \\
{\left[x_{-2}\right]_{\alpha} } & =\left[\frac{3}{2}+\alpha, 3-\frac{1}{2} \alpha\right] .
\end{aligned}
$$

From (81), it results in a coupled system of the difference equation with parameter $\alpha$, 


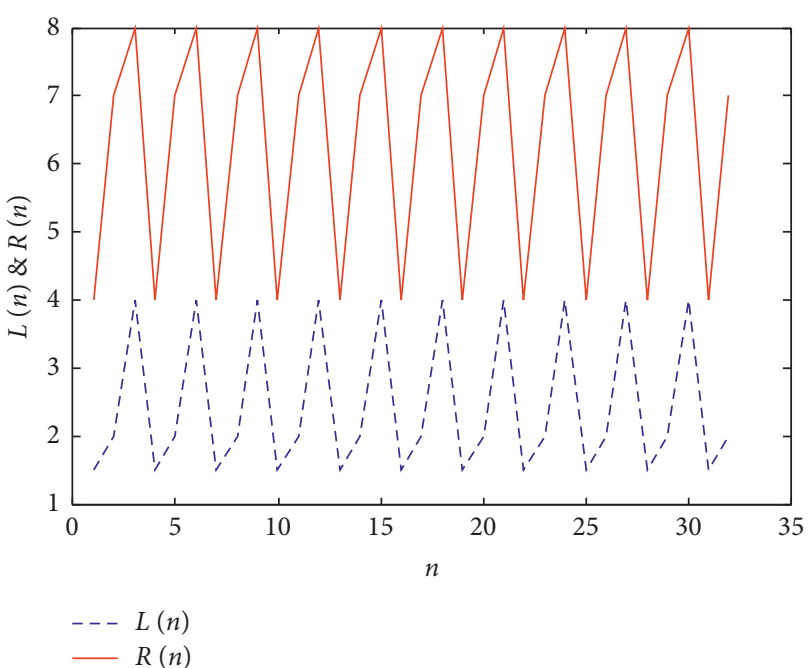

FIgURE 1: The solution of system (85) when $\alpha=0$.

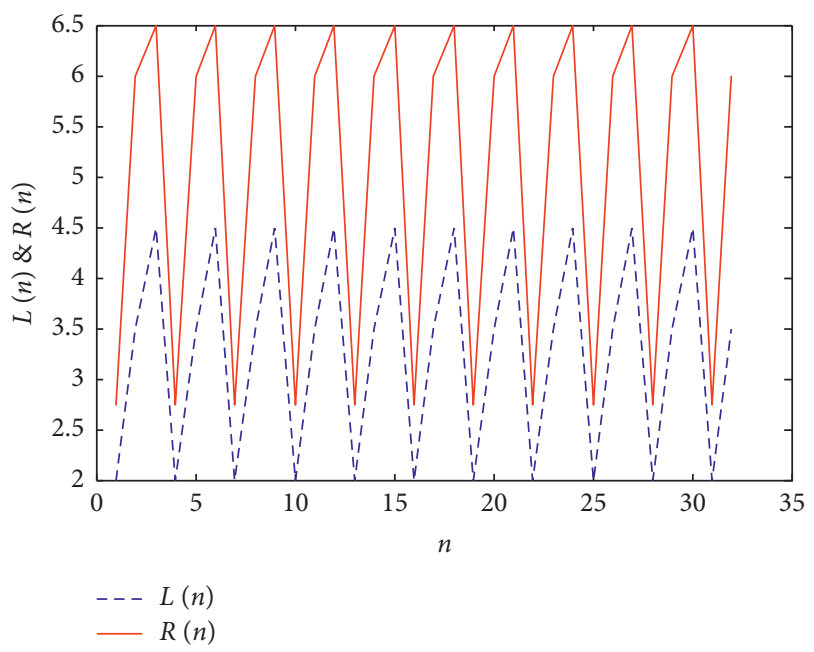

Figure 2: The solution of system (85) when $\alpha=0.5$.

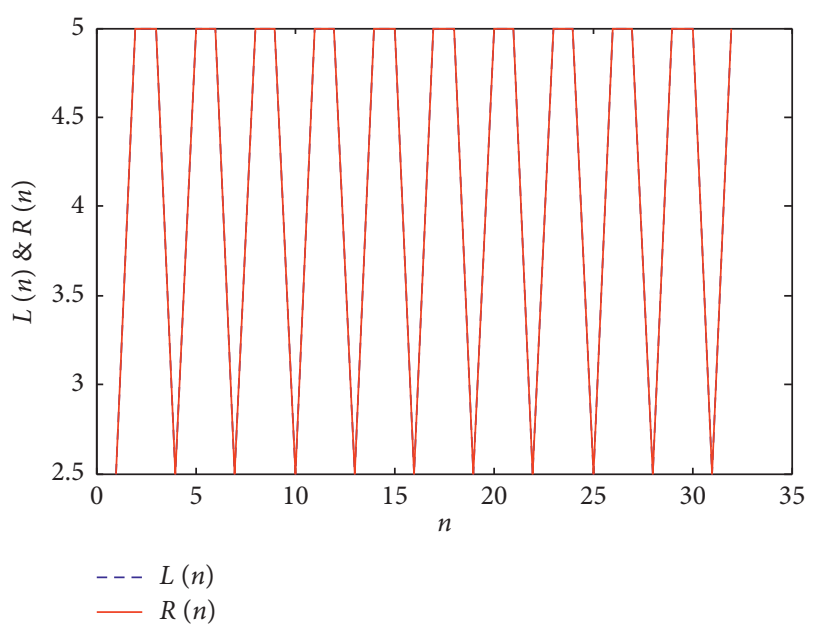

FIgURE 3: The solution of system (85) when $\alpha=1$.

$$
\begin{aligned}
& L_{n+1, \alpha}=\max \left\{\frac{3+2 \alpha}{R_{n, \alpha}}, \frac{3+2 \alpha}{R_{n-1, \alpha}}, L_{n-2, \alpha}\right\}, \\
& R_{n+1, \alpha}=\max \left\{\frac{8-3 \alpha}{L_{n, \alpha}}, \frac{8-3 \alpha}{L_{n-1, \alpha}}, R_{n-2, \alpha}\right\}, \\
& \alpha \in(0,1], n=0,1, \ldots
\end{aligned}
$$

It is easy to show that systems (81)-(83) satisfy the conditions of Theorem 3. It follows from Theorem 3 that every positive solution of systems (81)-(83) is eventually periodic with period three, bounded, and persists (see Figures 1-3).

\section{Data Availability}

No data were used to support this study.

\section{Conflicts of Interest}

The authors declare that they have no conflicts of interest.

\section{Authors' Contributions}

All authors contributed equally to each part of this work. All authors read and approved the final manuscript.

\section{Acknowledgments}

This work was supported by the Applied Basic Research Project of Sichuan Province (Grant nos. 2018JY0480 and 2018JY0169) of China.

\section{References}

[1] V. L. Kocic and G. Ladas, Global Behavior of Nonlinear Difference Equations of Higher Order with Applications, Kluwer Academic Publishers, Dordrecht, Netherlands, 1993.

[2] J. Migda, "Approximative solutions to difference equations of neutral type," Applied Mathematics and Computation, vol. 268, pp. 763-774, 2015.

[3] W. T. Jamieson and O. Merino, "Asymptotic behavior results for solutions to some nonlinear difference equations," Journal of Mathematical Analysis and Applications, vol. 430, no. 2, pp. 614-632, 2015.

[4] X. Liu, Y. Zhang, H. Shi, and X. Deng, "Existence of periodic solutions for a $2 \$ \$ n \$ \$ n$ th-order difference equation involving $\$ \$ p \$ \$ p$-laplacian," Bulletin of the Malaysian Mathematical Sciences Society, vol. 38, no. 3, pp. 1107-1125, 2015.

[5] E. P. Popov, Automatic Regulation and Control, Nauka, Moscow, Russia, 1966.

[6] Y. Gao and G. Zhang, "Oscillation of nonlinear first order neutral difference equations," Applied Mathematics E-Notes, vol. 1, pp. 5-10, 2001.

[7] J. Feuer, E. J. Janowski, G. Ladas, and C. Teixeira, "Global behavior of solutions of $x_{n+1}=\max \cdot\left\{x_{n}, A\right\} / x_{n} \cdot x_{n-1}$," Journal of Computational Analysis and Applications, vol. 2, no. 3, pp. 237-252, 2004.

[8] W. J. Briden, E. A. Grove, G. Ladas et al., "Eventually periodic solutions of $x_{n+1}=\max \left\{1 / x_{n}, A_{n} / x_{n-1}\right\}$." Communications on Applied Nonlinear Analysis, vol. 6, pp. 31-34, 1999. 
[9] G. Ladas, "Open problems and conjectures," Journal of Difference Equations and Applications, vol. 1, no. 1, pp. 95-97, 1995.

[10] W. T. Patula and H. D. Voulov, "On a max type recurrence relation with periodic coefficients," Journal of Difference Equations and Applications, vol. 10, no. 3, pp. 329-338, 2004.

[11] H. D. Voulov, "On the periodic character of some difference equations," Journal of Difference Equations and Applications, vol. 8, no. 9, pp. 799-810, 2002.

[12] A. M. Amleh, J. Hoag, and G. Ladas, "A difference equation with eventually periodic solutions," Computers \& Mathematics with Applications, vol. 36, no. 10-12, pp. 401-404, 1998.

[13] D. P. Mishev, W. T. Patula, and H. D. Voulov, "A reciprocal difference equation with maximum," Computers \& Mathematics with Applications, vol. 43, no. 8-9, pp. 1021-1026, 2002.

[14] A. Gelisken, C. Cinar, and I. Yalcinkaya, "On a max-type difference equation," Advances in Difference Equations, vol. 2010, no. 1, Article ID 584890, 2010.

[15] X. Yang, X. Liao, and C. Li, "On a difference equation with maximum," Applied Mathematics and Computation, vol. 181, no. 1, pp. 1-5, 2006.

[16] F. K. Sun, "On the asymptotic behavior of a difference equation with maximum," Discrete Dynamics in Nature and Society, vol. 2008, Article ID 243291, 6 pages, 2008.

[17] A. Gelisken and C. Cinar, "On the global attractivity of a maxtype difference equation," Discrete Dynamics in Nature and Society, vol. 2009, Article ID 812674, 5 pages, 2009.

[18] I. Szalkai, "On the periodicity of the sequence," Journal of Difference Equations and Applications, vol. 5, no. 1, pp. 25-29, 1999.

[19] J. Bidwell and J. E. Franke, "Bounded implies eventually periodic for the positive case of reciprocal-max difference equation with periodic parameters," Journal of Difference Equations and Applications, vol. 14, no. 3, pp. 321-326, 2008.

[20] D. W. Cranston and C. M. Kent, "On the boundedness of positive solutions of the reciprocal max-type difference equation $x_{n}=\max \left\{A_{n-1}^{1} / x_{n-1}, A_{n-1}^{2} / x_{n-2}, \ldots, A_{n-1}^{t} / x_{n-t}\right\}$ with periodic parameters," Applied Mathematics and Computation, vol. 221, pp. 144-151, 2013.

[21] B. Qin, T. X. Sun, and H. J. Xi, "Dynamics of the max-type difference equation $x_{n+1}=\max \left\{A / x_{n}, x_{n-k}\right\}$." Journal of Computational Analysis \& Applications, vol. 14, pp. 856-861, 2012.

[22] E. M. Elsayed and S. Stević, "On the max-type equation," Nonlinear Analysis: Theory, Methods \& Applications, vol. 71, no. 3-4, pp. 910-922, 2009.

[23] B. D. Iričanin and E. M. Elsayed, "On the max-type difference equation $x_{n+1}=\max \left\{A / x_{n}, x_{n-3}\right\}$." Discrete Dynamics in Nature and Society, vol. 2010, Article ID 675413, 13 pages, 2010.

[24] Q. Xiao and Q.-H. Shi, "Eventually periodic solutions of a max-type equation," Mathematical and Computer Modelling, vol. 57, no. 3-4, pp. 992-996, 2013.

[25] T. X. Sun, J. Liu, Q. L. He et al., "Eventually periodic solutions of a max-type difference equation," The Scientific World Journal, vol. 2014, Article ID 219437, 4 pages, 2014.

[26] P. Prasertsang and S. Sompong, "On the solutions of the maxtype difference equation $\left(\mathrm{x}_{-}\{\mathrm{k}+1\}\right)=\max \left\{\backslash\right.$ frac $\{1\}\left\{\mathrm{x}_{-}\{k-1\}\right.$ $\left.\left.\{n\}\}, \mathrm{x}_{-}\{k-1\} n\right\}\right\}, \mathrm{n} \backslash$ geq $1, "$ Applied Mathematical Sciences, vol. 7, pp. 1529-1535, 2013.

[27] K. S. Berenhaut, J. D. Foley, and S. Stević, "Boundedness character of positive solutions of a max difference equation," Journal of Difference Equations and Applications, vol. 12, no. 12, pp. 1193-1199, 2006.
[28] S. Stević, M. A. Alghamdi, A. Alotaibi et al., "Long-term behavior of positive solutions of a system of max-type difference equations," Applied Mathematics and Computation, vol. 235 , pp. 567-574, 2014.

[29] S. Stevic, M. A. Alghamdi, A. Alotaibi, and N. Shahzad, "Boundedness character of a max-type system of difference equations of second order," Electronic Journal of Qualitative Theory of Differential Equations, vol. 45, no. 45, pp. 1-12, 2014.

[30] S. Stević, "On a nonlinear generalized max-type difference equation," Journal of Mathematical Analysis and Applications, vol. 376, pp. 317-328, 2011.

[31] G. Papaschinopoulos and B. K. Papadopoulos, "On the fuzzy difference equation $x_{n}+1=A+x_{n} / x_{n}-m$," Fuzzy Sets and Systems, vol. 129, no. 1, pp. 73-81, 2002.

[32] Q. Zhang, L. Yang, and D. Liao, "On first order fuzzy ricatti difference equation," Information Sciences, vol. 270, pp. 226-236, 2014.

[33] Q. H. Zhang, J. Z. Liu, and Z. G. Luo, "Dynamical behavior of a third-order rational fuzzy difference equation," Advances in Difference Equations, vol. 2015, no. 1, 2015.

[34] Q. H. Zhang, L. H. Yang, and D. X. Liao, "Behavior of solutions to a fuzzy nonlinear difference equation," Iranian Journal of Fuzzy Systems, vol. 9, pp. 1-12, 2012.

[35] G. Papaschinopoulos and G. Stefanidou, "Boundedness and asymptotic behavior of the solutions of a fuzzy difference equation," Fuzzy Sets and Systems, vol. 140, no. 3, pp. 523-539, 2003.

[36] E. Y. Deeba, A. De Korvin, and E. L. Koh, "On a fuzzy logistic difference equation," Differential Equations and Dynamical Systems, vol. 4, pp. 149-156, 1996.

[37] E. Hatir, T. Mansour, and I. Yalcinkaya, "On a fuzzy difference equation,” Utilitas Mathematica, vol. 93, pp. 135-151, 2014.

[38] G. Papaschinopoulos and B. K. Papadopoulos, "On the fuzzy difference equation $\mathrm{xn}+1=\mathrm{A}+\mathrm{B} / \mathrm{xn}$," Soft Computing-A Fusion of Foundations, Methodologies and Applications, vol. 6, no. 6 , pp. $456-461,2002$.

[39] A. Khastan, "New solutions for first order linear fuzzy difference equations," Journal of Computational and Applied Mathematics, vol. 312, pp. 156-166, 2017.

[40] M. Pakdaman and S. Effati, "On fuzzy linear projection equation and applications," Fuzzy Optimization and Decision Making, vol. 15, no. 2, pp. 219-236, 2016.

[41] Q. L. He, C. Y. Tao, T. X. Sun et al., "Periodicity of the positive solutions of a fuzzy max-difference equation," Abstract and Applied Analysis, vol. 2014, Article ID 760247, 4 pages, 2014.

[42] G. Stefanidou and G. Papaschinopoulos, "Behavior of the positive solutions of fuzzy max-difference equations," $A d$ vances in Difference Equations, vol. 2, pp. 153-172, 2005.

[43] G. Stefanidou and G. Papaschinopoulos, "The periodic nature of the positive solutions of a nonlinear fuzzy max-difference equation," Information Sciences, vol. 176, no. 24, pp. 36943710, 2006.

[44] C. Wang, X. Su, P. Liu, X. Hu, and R. Li, "On the dynamics of a five-order fuzzy difference equation," The Journal of Nonlinear Sciences and Applications, vol. 10, no. 6, pp. 3303-3319, 2017.

[45] C. Wang, X. Jing, X. Hu, and R. Li, "On the periodicity of a max-type rational difference equation," The Journal of Nonlinear Sciences and Applications, vol. 10, no. 9, pp. 4648-4661, 2017.

[46] C. Y. Wang, X. J. Fang, and R. Li, "On the dynamics of a certain four-order fractional difference equations," Journal of 
Computational Analysis and Applications, vol. 22, pp. 968976, 2017.

[47] C. Y. Wang, Y. Q. Zhou, S. Pan, and R. Li, "On a system of three max-type nonlinear difference equations," Journal of Computational Analysis and Applications, vol. 25, pp. 14631479, 2018.

[48] L. L. Jia, "Dynamic behaviors of a class of high-order fuzzy difference equations," Journal of Mathematics, vol. 2020, Article ID 1737983, 13 pages, 2020.

[49] C. Y. Wang, J. H. Li, and L. L. Jia, "Dynamics of a high-order nonlinear fuzzy difference equation," Journal of Applied Analysis and Computation, vol. 2020, 2020 in Press. 